\title{
REPRESENTAÇÕES SOCIAIS DE CAMINHONEIROS DE ROTA CURTA
} SOBRE HIV/AIDS

\author{
REPRESENTACIONES SOCIALES DE LOS CONDUCTORES DE CAMIONES \\ SOBRE EL VIH/SIDA \\ SOCIAL REPRESENTATIONS OF SHORT DISTANCE TRUCK DRIVERS ABOUT \\ HIV/AIDS
}

\author{
Laio Magno Santos de Sousa \\ Universidade Federal da Bahia, Salvador/BA, Brasil \\ Lucineide Santos Silva \\ Universidade Federal do Vale do São Franscisco, Petrolina/PE, Brasil
}

Aline Tonheiro Palmeira

Universidade Federal da Bahia, Salvador/BA, Brasil

\begin{abstract}
RESUMO
Neste estudo qualitativo objetivou-se analisar representações sociais de caminhoneiros de rota curta sobre a AIDS, transmissão do HIV e prevenção da AIDS. Envolveu 19 caminhoneiros de rota curta, de uma associação em Vitória da Conquista-BA. A produção dos dados foi por intermédio de entrevista semiestruturada e por análise de conteúdo temática, sendo identificadas três categorias êmicas. Os resultados mostraram que as representações sociais da AIDS estavam ancoradas nos constructos: "doença ruim", "doença perigosa", "doença incurável" e "doença que mata". A transmissão do HIV relacionou-se ao sexo desprotegido com profissionais do sexo, gays e contato com sangue contaminado; a prevenção da AIDS foi representada pelo uso da camisinha nas relações extraconjugais e pela fidelidade da esposa. Apreendeu-se que as representações sobre AIDS estão embasadas em conhecimentos do início da epidemia e fundamentadas no distanciamento dos indivíduos, configurando quadro importante para a reflexão sobre o processo de vulnerabilização.
\end{abstract}

Palavras-chave: representação social; HIV/AIDS; sexualidade; vulnerabilidade; saúde do homem.

\section{RESUMEN}

Nuestro objetivo fue analizar las representaciones sociales de camioneros de ruta corta sobre el SIDA, la transmisión de VIH y la prevención del SIDA. La producción de datos fue por intermedio de entrevistas semi-estructuradas con 19 camioneros de una asociación de camioneros en Vitória da Conquista, Bahia. Por análisis de contenido temático se identificaron tres categorías émicas. Las representaciones sociales del SIDA estaban ancladas en los constructos: "enfermedad mala", "enfermedad incurable" y "enfermedad que mata". La transmisión del VIH se relacionó al sexo desprotegido con profesionales del sexo, gays y sangre contaminada. La prevención del SIDA fue representada por el uso de preservativos en las relaciones extraconyugales y fidelidad de la esposa. Las representaciones sobre el SIDA están ancladas en conocimientos desde el inicio de la epidemia y están fundadas en el distanciamiento de los individuos, configurando un marco importante para la reflexión sobre el proceso de vulnerabilización.

Palabras claves: representación social; VIH/SIDA; sexualidad; vulnerabilidad; salud del hombre.

\begin{abstract}
This qualitative study aimed to analyze social representations of short truck drivers about AIDS, HIV transmission and AIDS prevention. 19 truckers of an association of truck drivers in Vitória da Conquista, Bahia, were involved. Data production was through semi - structured interviews and thematic content analysis. Three emic categories were identified. The results showed that social representations of AIDS were anchored in these constructs: "bad disease"," dangerous disease ", "incurable disease" and "disease that kills". HIV transmission was related to unprotected sex with sex workers, gays and contaminated blood; AIDS prevention was represented by the use of condoms in extramarital relationships and fidelity of the wives. It was seized that the representations of AIDS are anchored in knowledge at the beginning of the epidemic and are based on the distance of individuals, configuring an important setting for reflection on the vulnerability processes.
\end{abstract}

Keywords: social representation; HIV/AIDS; sexuality; vulnerability; man's health. 


\section{Introdução}

O mundo presenciou, no início da década de 1980, o surgimento de uma doença que desafiou o campo científico, a Síndrome da Imunodeficiência Adquirida (AIDS), que logo se transformou em uma pandemia complexa, com especificidades tanto no âmbito biológico quanto no social. Naquela época, o anúncio dessa nova doença pelos meios de comunicação transmitiu informações do domínio médico e científico para o registro popular (Herzlich $\&$ Pierret, 2005). Foi possível acompanhar o desenvolvimento tanto médico como social da doença, assim como discutir os impactos que as estratégias de prevenção, cuidado e tratamento tiveram na população, e de que maneira as representações sociais sobre a AIDS foram sendo construídas (Herzlich \& Pierret, 2005; Jodelet, 2001; Sontag, 2007; Spink, Medrado, Menegon, Lyra, \& Lima, 2001).

A epidemia da AIDS ainda representa um grande desafio para a humanidade, pois adquiriu uma dinâmica peculiar, tornando-se um fenômeno global e distinto nas diversas regiões do mundo, sofrendo influências das práticas sociais dos seres humanos (Brito, Castilho, \& Szwarcwald, 2000). O Programa Conjunto das Nações Unidas sobre HIV/AIDS (Unaids, 2013) em seu último relatório, estima que cerca de 35,3 milhões de pessoas vivam atualmente com o vírus HIV em todo mundo. No Brasil, segundo dados do Sistema de Informação de Agravos de Notificação, existem 686.478 brasileiros com AIDS, dos quais $64,9 \%$ são do sexo masculino e $35,1 \%$ são do sexo feminino (Brasil, 2013).

No Brasil, a primeira década da epidemia ficou marcada pela restrição desta às áreas metropolitanas do Sudeste, aos homens que fazem sexo com homens, aos hemofílicos, aos que recebiam transfusão sanguínea e aos usuários de drogas injetáveis (Garcia \& Souza, 2010). No entanto, atualmente novos fenômenos têm transformado a face da epidemia no país: a heterossexualização, interiorização, pauperização e envelhecimento (Brito et al., 2000; Pinto et al., 2007). A epidemia encontra-se em expansão apenas nas regiões Norte e Nordeste, enquanto tem diminuído de forma marcante no Sudeste (Teixeira et al., 2014). Estes aspectos conferem à epidemia de AIDS características próprias, dinâmicas e, ao mesmo tempo, complexas, que exigem abordagens multidisciplinares, o que se constitui um desafio para os novos estudos referentes ao enfretamento ao HIV e à AIDS (Buchalla \& Paiva, 2002).

Neste contexto, alguns grupos sociais merecem atenção específica das ações de saúde, não no sentido de ressuscitar a antiga noção de grupos de risco, mas sim com o objetivo de observar questões que se tornam imprescindíveis para o planejamento e a implementação de políticas e programas voltados para os grupos que vivenciam contextos de maior vulnerabilidade (Garcia \& Souza, 2010). Nesse sentido, o Programa Conjunto das Nações Unidas sobre HIV/AIDS (UNAIDS, 2007) chama a atenção para alguns grupos que possuem vulnerabilidade acrescida para infecção pelo HIV, entre os quais estão os indivíduos que vivem em grande mobilidade, a exemplo dos caminhoneiros.

Em decorrência disso, múltiplos pesquisadores têm desenvolvido estudos que apontam para a intensidade dos contextos de vulnerabilidade vivenciados por caminhoneiros e destacam diversos aspectos no âmbito social, individual e programático envolvidos neste processo (Leal, 2008; Maason \& Monteiro, 2010; Nascimento, 2003; Rocha, 2008; Teles, 2008). No entanto, boa parte desses estudos têm se dedicado a investigar caminhoneiros que fazem rotas de longa distância, por conta da alta mobilidade vivenciada por estes sujeitos sem, contudo, focalizar especificamente os caminhoneiros de rota curta.

Estudo pioneiro realizado por Villarinho et al. (2002) indica a necessidade de estudos que levem em consideração a população de caminhoneiros de rota curta, pois a vulnerabilidade destes sujeitos não pode ser apenas associada ao tempo que permanecem fora de casa, mas também aos aspectos sociais e culturais mais amplos. No estudo realizado pelas autoras, com 269 indivíduos na área portuária de Santos, chama a atenção alguns aspectos sociais envolvidos na produção de vulnerabilidade destes caminhoneiros, sendo estes: a baixa adesão ao uso do preservativo, a cultura machista e a baixa percepção de vulnerabilidade ao HIV/AIDS. Além disso, $40 \%$ dos entrevistados referiram estar protegidos por conta da relação sexual com parceira fixa, apoiados na concepção de que existem parceiras sexuais mais seguras do que outras.

Nesse sentido, devem-se levar em consideração os aspectos relacionados à construção social das masculinidades, pois uma característica desta profissão a ser destacada é de que a maioria destes profissionais é do sexo masculino, contabilizando $98,2 \%$ da população total de caminhoneiros (IBGE, 2010). Pode-se ainda considerar a existência de influências de gênero na produção da vulnerabilidade destes indivíduos, principalmente na análise das posturas relacionadas ao padrão de masculinidade considerado hegemônico, que encara os riscos com naturalidade e o sexo como uma necessidade, um desejo incontrolável, no qual a "recusa de uma mulher" torna-se fato impensável (Koller, 2007). 
Dessa maneira, o conceito de masculinidades, situado no âmbito do gênero, pode ser fundamental para a compreensão das interações entre estes homens e as mulheres ao longo de suas trajetórias e casas. A masculinidade refere-se aos atributos, valores, funções e condutas esperados pelos homens de uma determinada cultura, sendo que este conjunto de questões varia ao longo do tempo e de acordo com outros marcadores sociais, como classe, raça, etc. (Gomes, 2008). As masculinidades são construídas ativamente no interagir dos sujeitos e envolve um complexo e continuado esforço de construção, sendo construídas, definidas e sustentadas coletivamente com base no consenso e não a partir do conflito direto (Connel e Lecture, 2002).

Dessa maneira, a partir do quadro de vulnerabilidade apresentado, este estudo pretendeu analisar as representações sociais de caminhoneiros de rota curta sobre a AIDS, transmissão do HIV e prevenção da doença, bem como analisar as implicações destas representações sobre a vulnerabilidade destes profissionais.

\section{As representações sociais e a vulnerabilidade ao HIV/AIDS}

A Teoria das Representações Sociais (TRS) foi desenvolvida a partir do conceito de representação coletiva cunhado por Emile Durkheim e repensado por Serge Moscovici (1978), para a sociedade do século XX, marcada pelo dinamismo e complexidade, surgindo a necessidade de um conceito que englobasse uma nova ordem de fenômenos (Sá, 1995). Moscovici conservou o conceito de representação e substituiu o conceito de "coletivo" (estático, positivista e cultural) pelo de "social", abarcando um sentido mais ampliado acerca do que é construído coletivamente (Guareshi, 1994). As representações sociais são compreendidas como "uma 'rede' de ideias, metáforas e imagens, mais ou menos interligadas livremente (...)" (Moscovici, 2003, p.210), surgindo como uma teoria que destaca o conhecimento produzido na interação humana (Moscovici, 1978).

Um dos propósitos das representações é fazer com que o não-familiar se torne familiar e este processo de familiarização é realizado por meio de dois processos interdependentes, que organizam as representações: a ancoragem e a objetivação (Moscovici, 2003). O primeiro se refere à maneira pela qual elementos estranhos e perturbadores de um sistema particular de categorias dos indivíduos são transformados e comparados com paradigmas de categorias supostamente apropriadas, sendo classificados e nomeados. Já o segundo processo revela a qualidade icônica de uma determinada ideia ou ser impreciso, reproduzindo em uma imagem concreta, um conceito abstrato, unindo a ideia de não-familiaridade com a de realidade (Moscovici, 2003).

As representações são entendidas como um conjunto de conhecimentos sociais, que possuem uma orientação prática e que permitem ao sujeito se situar no mundo e o dominar (Jodelet, 2001). No que se refere às funções, para Moscovici (2003), as representações sociais possuem, basicamente, duas: elas dão uma forma aos objetos, pessoas e acontecimentos a que se referem, localizando-os em uma determinada categoria que é compartilhada por um grupo de pessoas; e elas são prescritivas, uma vez que se impõem aos grupos por terem uma força estrutural e por estarem pautadas em uma tradição que as antecede.

Este objetivo prático da representação é importante no que diz respeito à contribuição "para os processos de formação de condutas e de orientação das comunicações sociais" (Moscovici, 1978, p. 77), o que pode ajudar na compreensão das estratégias adotadas pelos sujeitos diante da possibilidade de contaminação pelo HIV (Barbará; Sachetti \& Crepaldi, 2005) e, assim, esclarecer facetas da construção social e psicológica da vulnerabilidade dos sujeitos ao HIV para além de um prisma meramente comportamental e individual.

Neste contexto, o conceito de vulnerabilidade guarda possíveis interações com o conceito de representações sociais na medida em que não se centra em análises essencialmente comportamentais diferentemente do conceito de risco -, mas admite a análise das questões relacionadas à AIDS de maneira complexa, levando em consideração tanto aspectos individuais, quanto sociais e programáticos envolvidos no processo de suscetibilidade à enfermidade.

A emergência deste conceito inicia-se em 1989, sendo marcante principalmente pelo deslocamento das questões exclusivamente individuais (intrínseco aos grupos de risco) para a atenção aos aspectos sociais (Mann \& Tarantola, 1996). No início da epidemia, a compreensão do processo de contaminação e adoecimento foi construída a partir do marco conceitual do risco epidemiológico, que foi acusado posteriormente de reforçar processos de estigmatização através da caracterização de grupos de risco. Dessa maneira, foi cunhado conceito de comportamentos de risco, o que deslocou o foco de atenção dos grupos sociais para os comportamentos individuais de risco, porém ajudou no reforço da culpabilização dos sujeitos infectados pelo HIV (Ayres, Paiva, \& França-Jr, 2012). 
A epidemia de AIDS mostrou que não há um único modo de descrição dos determinantes, distribuição e desdobramentos de um processo de saúde-doença-cuidado, mas levantou questões ainda mais contundentes no sentido de que o processo saúde-doença é mediado por uma diversidade de determinantes e condicionantes que vão desde o indivíduo enquanto sujeito e a partir de suas intersubjetividades, à sociedade como um todo, isto é, os aspectos macroestruturais que influenciam o processo de adoecimento. Não existe um agente agressor em si que seja responsável pela produção da doença, mas diversos agentes que dependem do ambiente cultural e sociopolítico, bem como do contexto intersubjetivo das pessoas (Ayres et al, 2012) Nesta perspectiva, as representações sociais fazem-se necessárias para a análise da vulnerabilidade.

\section{Metodologia}

\section{Trabalho de campo e sujeitos do estudo}

A pesquisa foi realizada entre os meses de janeiro e fevereiro de 2011 em Vitória da Conquista, cidade localizada na Região Sudoeste da Bahia, que possui uma população de 306.866 habitantes (IBGE, 2012) e é cortada pela BR 116, com grande trânsito de pessoas e de mercadorias. Antes de iniciar o campo, foram realizadas duas entrevistas piloto (que não entraram na análise dos dados) com o objetivo de testar o roteiro.

O trabalho de campo foi realizado numa pequena associação de caminhoneiros localizada no centro da cidade, onde os profissionais se aglomeram na espera da contratação de pequenos "fretes" para cidades vizinhas. Este é um espaço dedicado ao trabalho, mas também à intensa sociabilidade entre os caminhoneiros e outras pessoas. A grande maioria dos sujeitos desta associação realizavam rotas curtas, o que colaborou para que todos os participantes desta investigação tivessem este perfil.

A quantidade de colaboradores recrutados foi de acordo com o critério de saturação das entrevistas (Minayo, 2007). Ao todo foram entrevistados 19 caminhoneiros, os quais foram incluídos no estudo a partir dos seguintes critérios: ser do sexo masculino, com idade igual ou superior a 18 anos, possuir no mínimo um ano de trabalho como caminhoneiro, bem como a assinatura do Termo de Consentimento Livre e Esclarecido (TCLE). Apesar de muitos destes profissionais já terem trabalhado com viagens de longa distância, no momento da pesquisa, estes sujeitos relataram percorrer pequenas distâncias, geralmente realizando viagens para entrega de pequenos fretes na própria cidade ou nos municípios vizinhos.

\section{Técnica de produção e análise de dados}

Trata-se de um estudo qualitativo. Para produção dos dados utilizou-se a entrevista semiestruturada, com o auxilio de um roteiro temático pré-estabelecido e gravador de áudio. As entrevistas aconteceram em sala restrita, dentro da associação de caminhoneiros, como forma de garantir o sigilo e a espontaneidade da entrevista.

As entrevistas foram transcritas integralmente e organizadas em núcleos temáticos a partir da Análise de Conteúdo proposta por Bardin (1977). As entrevistas foram lidas exaustivamente na íntegra, depois foram registrados os temas presentes ao longo delas, para, por fim, serem identificados os eixos temáticos que condensavam as representações sociais sobre HIV/AIDS. Assim, as três categorias êmicas foram: "Representações insistentes acerca da AIDS: 'doença perigosa, ruim, incurável e que leva a morte"', "Transmissão da AIDS: 'o perigo está no sexo e no sangue!" e "Prevenção da AIDS: "é melhor usar camisinha e ser fiel!"”. Essas categorias trouxeram elementos de ancoragem e objetivação, pressupostos da Teoria das Representações Sociais, o que resultou na discussão dos dados nessa perspectiva, identificando representações que podem interferir no processo de vulnerabilização ao HIV.

\section{Aspectos éticos}

Todas as fases desta pesquisa foram realizadas em conformidade ao que propõe a Resolução $n^{\circ}$ 466/2012 (Conselho Nacional de Saúde, 2012), ou seja, todos os participantes assinaram o TCLE. O projeto foi aprovado pelo Comitê de Ética em Pesquisa (CEP) da Universidade Estadual do Sudoeste da Bahia, sob o número de protocolo 208/2010 e CAE 0044.0.454.000-10.

\section{Resultados e discussão}

Representações insistentes: doença perigosa, ruim, incurável e que leva a morte

Como poderemos observar mais detalhadamente na Tabela 1 , os sujeitos da pesquisa eram, em sua maioria, casados ou em vivência de união estável. A idade deles variou entre 24 e 65 anos (com média de 50,3 anos). Em relação ao tempo de profissão como caminhoneiros, a média entre esses indivíduos foi de 24,8 anos. 
Para os sujeitos, HIV/AIDS estava associada ao status de "doença ruim" e "perigosa", principalmente pelo seu caráter incurável ("doença incurável”) e, por conseguinte, por sua relação com a morte ("doença que mata"). Tais representações estão ancoradas historicamente na construção social da AIDS, que traz, desde a sua origem, a relação com o sofrimento e a finitude. Isso pôde ser identificado no seguinte trecho da entrevista: "[a AIDS] é uma doença terrível, ... ela é incurável... Pra mim é um terror. Não há outra coisa na vida mais ruim do que isso... Vai levar pra o abismo, vai levar a vida a óbito." (E4, 55 anos).

Tabela 1. Caracterização sociodemográfica dos colaboradores do estudo, Vitória da Conquista, 2011

\begin{tabular}{cc}
\hline \multicolumn{1}{c}{ Características } & $\mathbf{n}$ \\
\hline Idade (anos) & 3 \\
$24-40$ anos & 11 \\
$41-59$ anos & 5 \\
$>60$ anos &
\end{tabular}

Situação conjugal

Com parceira (casado ou

em união estável)

Sem parceira (solteiro)

Nível educacional

Ensino fundamental incompleto

Ensino fundamental completo

Ensino médio (completo e incompleto)

Experiência como caminhoneiro (em anos)

$$
2-20 \text {. }
$$

$>21$

\begin{tabular}{lc} 
Religião & \\
Católico & 15 \\
Evangélico & 3 \\
Não possui & 1 \\
\hline
\end{tabular}

Diversos estudos trazem a morte como representação relacionada ao HIV/AIDS, como as pesquisas com grupos de adolescentes (Camargo, Bertoldo, \& Barbará, 2009; Thiengo et al., 2005), com homens rurais (Alves, 2003), com trabalhadores (Marques, Gomes, \& Oliveira, 2004) e com pacientes soropositivos em tratamento (Torres \& Camargo, 2008), o que nos faz perceber que esta representação está fortemente presente em diversos grupos etários e sociais. O HIV/AIDS associado à ideia de morte dessa forma tão irrestrita, ou seja, para homens, mulheres, jovens, pessoas mais velhas e mesmo para pessoas que estão em tratamento, aponta para a força da ancoragem nesse elemento representacional.

Segundo Marques et al. (2004), esta representação torna evidente uma dimensão imagética, que expressa o sentimento de destruição frente a AIDS e tem sua gênese fundamentada nas primeiras informações divulgadas pela mídia e pelos estudos científicos no início da epidemia. De acordo com esses autores, o motivo da permanência desta representação é que a AIDS continua sendo uma doença incurável, mesmo após os grandes avanços científicos que prolongam, efetivamente, a vida dos portadores, mas não os curam.

Nesta perspectiva, a representação de doença incurável foi identificada e pesquisas envolvendo outros grupos sociais (Alves, 2003; Camargo et al., 2009; Thiengo et al., 2005; Torres \& Camargo, 2008) e também no presente estudo, podendo ser destacada nesse fragmento:

É uma doença que não tem cura, né!... Ele pode viver um tempo, mas um dia ele vai ter que ir. Claro que nós todos um dia vamos. Mas ali ele já sabe que tem uma certeza, né!... a preocupação que eu tenho é que geralmente é uma doença que não tem cura, né! (E12, 50 anos)

Neste trecho, para o entrevistado, AIDS é uma doença que tem sua presença de maneira marcante, além de ter sua imagem representada pela morte. Também é possível interpretar que, a partir do momento em que a pessoa passa a ter esse diagnóstico, não é possível reverte-lo e que a morte vai ocorrer por causa do adoecimento: "ele vai ter que ir". Nesse sentido, nessa representação, morrer é uma questão de tempo e a vida que se tem é marcada pela presença da doença.

Assim, outro elemento da representação social da AIDS é o de doença como sendo ruim, que também foi encontrado em outras pesquisas (Thiengo et al., 2005) e tal resultado sugere uma associação ao sofrimento físico (emagrecimento, déficit na aparência visual, invalidez, dentre outros) e psíquico (preconceito, depressão, estar com uma doença incurável) provocados pelas co-infecções e divulgadas no início da epidemia, como ilustra o fragmento a seguir: 
Eu já vi o cara morrendo de AIDS. Morreu lá muito feio, magrelinho, ai o povo dizia que era AIDS. Foi logo quando começou esse negócio de AIDS, ai foi que eu tomei medo. Ai eu tomei medo mesmo! O cara ficou careca. Ali sofreu, porque se morresse logo num tinha problema, mas não ficou muito tempo na cama. Isso tem uns 20 anos. (E16, 54 anos)

Desse modo, tendo em vista o sofrimento físico e psíquico provocado pela AIDS, aliado à possibilidade iminente de morte, a representação de doença "perigosa" foi identificada neste estudo e também em outra pesquisa (Camargo et al, 2009). Pode-se representá-la no seguinte relato:

\section{A AIDS é uma doença muito perigosa que não tem cura. (...) você pegou, você teve AIDS! Você pode prolongar a vida, mas você num tem cura. Você vai morrer dela, num tem jeito. (E13, 63 anos)}

As representações, como uma forma de conhecimento e domínio sobre o mundo, possuem uma orientação prática e permitem ao sujeito se situar no mundo e o dominar (Almeida, 2005). Dessa forma, podem interferir, junto com outros elementos, de modo a contribuir na intensificação do processo de vulnerabilização dos caminhoneiros. Nesse sentido, apesar de terem representado a AIDS como uma doença perigosa, incurável, ruim e que leva à morte, estes sujeitos puderam assumir certa negação no risco ao HIV, o que foi encontrado principalmente quando indagados a respeito da possibilidade de infecção deles pelo vírus.

Além disso, apesar da eficiência do tratamento da doença que transformou o perfil de sua evolução clínico-epidemiológica, caracterizando-a como uma doença crônica, de baixa letalidade e com elevada sobrevida dos indivíduos portadores, percebe-se que as representações sociais dos caminhoneiros insistem em categorias de outrora.

\section{Transmissão da AIDS: "o perigo está no sexo e no sangue!”}

Noque dizrespeitoàs representações sociais sobre as formas de transmissão da AIDS, os caminhoneiros declararam que a relação sexual desprotegida e o sangue contaminado (através de pérfuro-cortantes, materiais hospitalares ou transfusão de sangue) são os principais veículos para a transmissão do vírus HIV. Outros estudos realizados com caminhoneiros também demonstraram que a transmissão do HIV foi reconhecida através do ato sexual sem preservativo, corroborando com os atuais achados (Rocha, 2008; Nascimento, 2003). Além disso, compreensões não científicas quanto à transmissão da doença também foram identificadas nos discursos, tais como picadas de insetos, beijo na boca e toalhas de banho:

Eu acho que ele pode ser transmitido pela saliva né? Pelo sangue... Uma coisa assim né? ... Assim... Uma toalha ou umas coisas que a pessoa tem contato assim de saliva ou de sangue, essas coisas, uma seringa né? (E12, 50 anos)

Um aspecto contraditório importante a ser ressaltado é o fato de que, mesmo as falas dos sujeitos estando em torno da transmissão da AIDS através do sexo, esta prática não se referia à realizada com as esposas, por exemplo, mas com as pessoas sob maior risco entre as quais estariam os profissionais do sexo (homens, mulheres e travestis) e homens que fazem sexo com outros homens, como pode ser observado no relato a seguir:

A forma de transmissão é... praticando sexo, né? Com essas pessoas... homossexual, essas coisas, né? Sem camisinha... ai deve pegar, né?... É através do homossexual, de uma prostituta, de uma pessoa já afetada, é desse tipo aí. (E1, 55 anos)

Nesse sentido, percebe-se que os clássicos grupos de risco, divulgados amplamente no início da epidemia, são imagens e significados recuperados por estes sujeitos. Desse modo, faz-se importante reportar à pesquisa de Herzlich e Pierret (2005, p. 75) que, ao investigarem notícias sobre AIDS veiculadas em jornais franceses do início da década de 1980, constataram que até julho de 1983 todos os termos empregados faziam certa "referência ao grupo mais frequentemente associado à doença, os homossexuais: 'pneumonia dos homossexuais', 'câncer dos homossexuais' ou 'câncer gay' e, mais amplamente, 'síndrome dos homossexuais' ou 'síndrome gay"'.

Desse modo, o simples passar do tempo não é a única condição para a mudança em uma representação social e as modificações que ocorrem em torno de um fenômeno precisam alcançar as mais diversas esferas (sociais, culturais e, por vezes, históricas) para que possam efetivar uma mudança significativa em sua representação. Essa condição de mudança é igualmente discutida por Moscovici (2003) "Uma vez criadas, contudo, elas [representações sociais] adquirem uma vida própria, circulam, se encontram, se atraem e se repelem e dão oportunidade ao nascimento de novas representações, enquanto velhas representações morrem" (p. 41), e todo esse processo é construído em um amplo espectro de fenômenos sociais .

No que tange à associação da AIDS com as profissionais do sexo, fica clara a relação da doença com os comportamentos sexuais que se desviam das concepções dominantes de gênero, o que pode 
revelar um pensamento de que, para estes sujeitos, as profissionais do sexo são disseminadoras do HIV, uma vez que adotam comportamento social e sexual distinto do esperado para as "mulheres de casa".

Embora os sujeitos reconheçam o sexo desprotegido com profissionais do sexo como sendo um comportamento de risco, aqui o sujeito vulnerável não são eles mesmos, mas os "outros" caminhoneiros, principalmente aqueles que realizam rotas de longas distâncias. Esta prática de exposição era citada como distante deles, enquanto indivíduos e grupo social específico (caminhoneiros de rota curta da associação).

esse grupo daqui é um grupo sossegado! Agora os [caminhoneiros] de estrada [rota longa] eles vivem muito sozinhos, só viajando, só viajando... (E8, 52 anos)

Nesse trecho, possivelmente esteja presente a percepção da AIDS enquanto "doença do outro", conteúdo comumente encontrado em estudos de AIDS que trabalham com esta mesma abordagem teórica (Alves, 2003; Joffe, 1995; Oltramari \& Camargo, 2004; Thiengo et al., 2005). Essa representação da AIDS traz consequências nocivas no que tange à vulnerabilização dos caminhoneiros à infecção, produzindo uma postura de "invulnerabilidade" frente à epidemia. Além disso, esta concepção pode reafirmar o preconceito contra grupos historicamente estereotipados, como assinala Joffe $(1995$, p. 318): "as representações sociais que constroem o 'outro' como aberração têm consequências para a prática. Elas permitem que esse 'outro' seja maltratado e discriminado".

A representação social da transmissão da AIDS, associada ao sangue contaminado, foi relatada pelos caminhoneiros, com ênfase nos materiais pérfurocortantes, materiais hospitalares e transfusão de sangue.

eu fiz cirurgias, e eu já ia assombrado! Meu problema não era morrer na banca da cirurgia, era sair de lá com uma epidemia dessas, com uma droga dessas, com uma circunstância dessa no sangue. Eu já vi, não foi o primeiro nem o último a acontecer... Aqui em Vitória da Conquista mesmo já houve casos desses aqui, entendeu? Às vezes num posto médico, se eu for fazer uma vacina, qualquer coisa, ou fazer um exame de sangue num laboratório, eu procuro logo ver o material que eles estão usando. (E4, 55 anos)

Aqui, deve-se considerar tanto as informações divulgadas por programas governamentais, quanto a rotina das estradas com a consequente exposição aos acidentes de trânsito e, por sua vez, aos distintos procedimentos hospitalares, como possíveis elementos que chamam atenção para a vulnerabilidade relacionada à profissão de caminhoneiro.

\section{Prevenção da AIDS: "é melhor usar camisinha e ser fiel!"}

As representações sociais relativas à prevenção da AIDS estiveram relacionadas principalmente ao uso da camisinha, ao casamento e à fidelidade. No que diz respeito ao uso do preservativo, os caminhoneiros relataram baixa adesão nas relações sexuais com parceira fixa. Ressaltaram que, neste caso, o uso da camisinha está relacionado à necessidade de contracepção, como é possível verificar a seguir:

\begin{abstract}
No casamento, eu nunca usei camisinha no casamento não. ... eu tive relação com ela, antes de casar, aí teve algumas vezes que eu usei camisinha, mas num era por questão de doença, mas era por questão de num ter filho, por não querer ter filho, mas não por questão de doença, entendeu? (E11, 49 anos)
\end{abstract}

Outros estudos corroboram com o achado (Dude et al., 2010; Leal, 2008; Nascimento, 2003; Rocha, 2008; Villarinho et al., 2002) e podem ser considerados comportamentos individuais importantes para a vulnerabilidade dos sujeitos ao HIV/AIDS na medida em que não usar preservativo com parceira fixa pode aumentar a exposição ao vírus. Um estudo epidemiológico mostrou a tendência da associação entre o não uso do preservativo com parceiras fixas e as doenças sexualmente transmissíveis (Teles et al., 2008). O uso do preservativo nas relações com parceiras fixas é extensamente relacionado à infidelidade, fazendo com que a sua utilização em relacionamentos conjugais seja de baixa adesão (Guerriero et al., 2002; Madureira \& Trentini, 2008).

Por outro lado, o uso do preservativo pelos caminhoneiros nas relações extra-conjugais demonstrou ser mais efetivo entre os caminhoneiros estudados, tal qual foi observado em outros estudos envolvendo homens (Guerriero et al., 2002; Madureira \& Trentini, 2008; Silva, 2002). Segundo Silva (2002), o uso da camisinha está relacionado às relações sexuais com pessoas "desconhecidas", fato que caracteriza a existência de um pensamento "mágico", que traz o entendimento de que a aparência e o pseudoconhecimento do perfil comportamental da outra pessoa possam garantir a proteção à AIDS. Por outro lado, Maia et al. (2008) acrescentam que a relação histórica do uso do preservativo com a prostituição e as relações extraconjugais podem inibir a incorporação deste hábito no território do casamento.

Malta et al. (2006) constataram, em seu estudo, que quanto mais os caminhoneiros tinham intimidade 
com as parceiras sexuais, menos eles usavam o preservativo de forma consistente. Outras crenças e percepções estão envolvidas também nesse processo, como a crença de que o uso do preservativo pode afetar a ereção (Guerriero et al., 2002; Madureira \& Trentini, 2008), o prazer, a estética e o desempenho sexual masculino (Guerriero et al., 2002; Madureira \& Trentini, 2008). Rebello et al. (2011) destacam que o preservativo masculino pode, simbolicamente, afetar atributos hegemônicos do modelo ideal do que é ser homem. No nosso estudo, os caminhoneiros enfatizaram, principalmente, a diminuição do prazer, provocada pela ausência de contato direto do pênis, como se pode verificar a seguir:

Eu não acho bom porque ela dá uma sensação de você está com uma luva. É como você botar uma luva na mão, aí você fica sem aquele contato, aquele contato natural, né? Então a camisinha tira aquele contato, aquela sensibilidade. Ai é por isso que eu não gosto. Eu já usei, mas não gosto não. (E12, 49 anos)

O uso não sistemático do preservativo é apontado, dentre tantos outros motivos, como uma das causas para o aumento progressivo da epidemia de AIDS em indivíduos com idade superior a 50 anos (Pottes et al., 2007). Os sujeitos deste estudo (média de 50,3 anos) corroboram com esse panorama e confirmam a importância de serem considerados aspectos geracionais na análise da vulnerabilidade. Rocha (2008), em pesquisa com caminhoneiros em regiões de fronteira de Rondônia, revelou que os mais jovens estavam mais sensibilizados com a ideia de prevenção, sobretudo em decorrência do apelo ao uso do preservativo, evidenciado desde a sua juventude até os dias atuais. De modo contrário e pela mesma razão, os homens com idade mais avançada não incorporaram a necessidade do seu uso, sendo difícil inserir o uso do preservativo nas relações sexuais após a maturidade.

Leal (2008) enfatiza que esta variável também pode estar relacionada com a baixa escolaridade, pois, na sua pesquisa, na medida em que a idade dos caminhoneiros aumentava, os níveis de escolaridade diminuíam numa relação inversamente proporcional. Nessa perspectiva, a baixa escolaridade apresentada pelos sujeitos deste estudo (79\% dos entrevistados têm até o ensino fundamental completo) pode implicar na dificuldade de acesso a informações adequadas e atualizadas sobre a AIDS. Outros estudos realizados com caminhoneiros também identificaram semelhante resultado (Leal, 2008; Masson \& Monteiro, 2010; Nascimento, 2003; Rocha, 2008, Teles, 2008; Villarinho, 2002).

Diante desses achados, verifica-se que embora os sujeitos do estudo, tenham ancorado que o uso do preservativo nas relações sexuais seja efetivo para a prevenção do HIV, diversos fatores socioculturais contribuem para que eles coloquem em prática uma ação diferente, isto é, tornando em algumas situações o uso do preservativo como algo dispensável.

Outras representações, apreendidas nos discurso dos caminhoneiros acerca da prevenção da AIDS, foram o "casamento" e a "fidelidade". Embora estas representações possam ser percebidas enquanto sinônimos, neste estudo foram identificadas conotações distintas para as mesmas. De acordo com os sujeitos, o casamento não implica, necessariamente, em comportamento monogâmico para a maioria dos homens, mas preserva a confiança de que as mulheres casadas são fiéis e sempre monogâmicas. A percepção de invulnerabilidade aqui pode estar associada à expectativa de fidelidade da esposa, como se pode observar na seguinte expressão:

como eu sou casado, faço sexo com a minha esposa, né! Se não for com ela... se acontecer com outra pessoa... tem que ser com preservativo! Com a esposa não precisa de preservativo pelo motivo de segurança, né, pois é uma pessoa que você conhece, tá vivendo junto há muito tempo, pela confiança que você tem nessa pessoa também. (E5, 28 anos)

Neste contexto, pode-se destacar a influência da concepção hegemônica de masculinidade na construção da vulnerabilidade destes caminhoneiros que, não tão raro, motivam os homens a assumirem alguns comportamentos de risco para provar sua masculinidade. Nessa perspectiva, Guerriero, Ayres e Hearst (2002) sinalizam que muitos homens tendem a não se perceberem vulneráveis ao HIV quando confiam na fidelidade da esposa, quando diminuem o número de parceiras ou quando se julgam capazes de selecioná-las criteriosamente. Tais dados apontam para análise de questões de gênero associadas ao modelo normativo ou hegemônico de masculinidade, que pode resultar em males para a saúde dos homens.

\section{Considerações finais}

O estudo acerca das representações sociais de caminhoneiros sobre a AIDS abre a possibilidade de nos aproximarmos das maneiras pelas quais estes sujeitos representam uma "realidade" que ao mesmo tempo pode ser considerada próxima e distante. A doença em tela foi fundamentada em aspectos "negativos" e, continuamente, representada de forma distanciada como “doença do outro". Já as representações a respeito de sua prevenção e sua transmissão, encontraram-se ancoradas em conhecimentos divulgados amplamente 
no início da epidemia, mas também relacionados com a rotina que envolve o ser caminhoneiro: viagens, acidentes em estradas, sexo desprotegido com outros parceiros que não os habituais.

ATRS não tem como fundamento fazer previsões de comportamento baseadas nas representações identificadas, mas o próprio Moscovici (2003) chamou atenção para a função prescritiva que as representações sociais podem ter, nos ajudando a pensar que arranjos são possíveis a partir delas. As representações são fruto dessa rede de significados que, por sua vez, não se constitui desarticulada de uma prática. Inserir estudos sobre representações de HIV/AIDS, justamente por contemplarem dos aspectos mais idiossincráticos aos mais sociais na construção de um significado e de uma prática, em políticas voltadas para o cuidado em saúde parece confirmar uma necessidade: a de que os sujeitos, com suas histórias e realidades, possam ser contemplados nos programas e ações de saúde.

Dessa maneira, as representações aqui discutidas acrescentam elementos importantes para a reflexão tanto de questões relacionadas à TRS, quanto sobre processos de vulnerabilização ao HIV vivenciados pelos caminhoneiros de rota curta. E apontam também para a necessidade de novos estudos que aprofundem investigações a respeito de como estes sujeitos representam elementos relacionados às suas próprias masculinidades e sexualidades, levando em consideração processos intersubjetivos e questões de gênero.

\section{Referências}

Almeida, A. M. O. (2005). A pesquisa em representações sociais: proposições teórico-metodológicas. In: M. F. S. Santos \& L. M. Almeida (Orgs.), Diálogos com a teoria das representações sociais. Recife: Editora Universitária da UFPE.

Ayres, J. R. C. M., Paiva V., \& França Jr I. F. (2012). Conceitos e práticas de prevenção: da história natural da doença ao quadro da vulnerabilidade e direitos humanos. In V. Paiva, J. R. C. M. Ayres, \& C. M. Buchalla. (Orgs), Vulnerabilidade e direitos humanos: prevenção e promoção da saúde: livro I, da doença à cidadania (pp. 71-94). Curitiba: Juruá.

Alves, M. D. F. P. (2003). Sexualidade e prevenção de DST/ AIDS: representações sociais de homens rurais de um município da zona da mata pernambucana, Brasil. Cadernos Saúde Pública, 19 (2), 429-439.

Barbará, A., Sachetti, V. A. R,. \& Crepaldi, M. A. (2005). Contribuições das representações sociais ao estudo da aids. Interação em Psicologia, 9(2), 331-339.

Bardin, L. (1977). Análise de Conteúdo. Lisboa: Edições 70.

Brito, A. M., Castilho, E. A., \& Szwarcwald, C.L. (2000). AIDS e infecção pelo HIV no Brasil: uma epidemia multifacetada. Rev Soc Bras Med Trop, 34(2), 207-217.

Camargo, V. B., Bertoldo, R. B., \& Barbará, A. (2009). Representações sociais da AIDS e alteridade. Estudos e Pesquisas em Psicologia, 9(3), 710-723.
Connel, R. W. (2002). Understanding men: gender sociology and the newinternational research on masculinities. Social Thought \& Research, 24, 13-31.

Conselho Nacional de Saúde - CNS. (2012). Resolução 466, de 12 de dezembro de 2012. Brasília: Autor.

Dude, A., Oruganti, G., Kumar, V., Mayer, K. H., Yeldandi, V., \& Schneider, J. A. (2009). HIV infection, genital symptoms and sexual risk behavior among indian truck drivers from a large transportation company in South Índia. Journal of Global Infectious Diseases, 1(1), 21-28.

Garcia, S. \& Souza, F.M.D. (2010). Vulnerabilidades ao HIV/ aids no contexto brasileiro: iniquidades de gênero, raça e geração. Saúde e sociedade, 19(Supl 2), 9-20.

Gomes, R. \& Nascimento, E.F.D. (2006) A produção do conhecimento da saúde pública sobre a relação homemsaúde: uma revisão bibliográfica. Cadernos de Saúde Pública, 22(5), 901-911.

Herzlich, C. \& Pierret, J. (2005). Uma doença no espaço público: a AIDS em seis jornais franceses. Physis, 15(Supl.), 71-101.

Instituto Brasileiro de Geografia e Estatística (IBGE). (2010) Censo demográfico, 2000 Acesso em 06 de janeiro, 2014, em http://www.sidra.ibge.gov.br/bda/tabela/protabl. asp? $c=3594 \& z=c d \& 0=17 \& i=P$

Instituto Brasileiro de Geografia e Estatística (IBGE). (2010) Ibge Cidades@ Acesso em 16 de junho, 2010 em: http:// www.ibge.gov.br/cidadesat/topwindow.htm?1

Jodelet, D. (2001). Representações sociais: um domínio em expansão. In D. Jodelet (Org.), As representações sociais (pp. 17-44). Rio de Janeiro: EdUERJ.

Joffe, H. (1994). "Eu não", "O meu grupo não": representações sociais transculturais da AIDS. In P. A. Guareschi \& S. Jovchelovitch (Orgs.), Textos em Representações sociais, (pp. 297-323). Petrópolis: Vozes.

Guerriero, I., Ayres, J. R. C. M., \& Hearst, N. (2002); Masculinidade e vulnerabilidade ao HIV de homens heterossexuais, São Paulo, SP. Revista de Saúde Pública, 36(4), 50-60.

Koller, E. M. P. (2007). Da vulnerabilidade ao protagonismo: profissionais do sexo e caminhoneiros frente à AIDS. Tese de Doutorado, Programa de Pós-Graduação em Enfermagem, Universidade Federal de Santa Catarina, Florianópolis, SC.

Leal, A. F. (2008). "No peito e na raça": a construção da vulnerabilidade de caminhoneiros: um estudo antropológico de políticas públicas para HIV/AIDS no Sul do Brasil. Tese de Doutorado, Programa de Pós-Graduação em Antropologia Social, Universidade Federal do Rio Grande do Sul, Porto Alegre, RS.

Marques, S. C., Oliveira, D. C. D., \& Gomes, A. M. T. (2004). AIDS e representações sociais: uma análise comparativa entre subgrupos de trabalhadores. Psicologia: Teoria $e$ Prática, edição especial, 91-104.

Madureira, V. S. F. \& Trentini, M. (2008). Da utilização do preservativo masculino à prevenção de DST/AIDS. Revista Ciência e Saúde Coletiva, 13(6), 1807-1816.

Maia, C., Guilhem, D., \& Freitas, D. (2008). Vulnerabilidade ao HIV/AIDS de pessoas heterossexuais casadas ou em união estável. Revista de Saúde Pública, 42(2), 242-248.

Malta, M., Bastos, F. I., Pereira-Koller, E. M., Cunha, M. D., Marques, C., \& Strathdee, S. A. (2006). A qualitative assessment of long distance truck drivers' vulnerability to HIV/ AIDS in Itajaí, southern Brazil. AIDS Care, 18(5), 489-496.

Masson, V. A. \& Monteiro, M.I. (2010). Vulnerabilidade à doenças sexualmente transmissíves/AIDS e uso de drogas 
psicoativas por caminhoneiros. Revista Brasileira de Enfermagem, 63(1), 79-83.

Minayo, M. C. S. (2007). O desafio do conhecimento: pesquisa qualitativa em saúde. São Paulo: Hucitec.

Ministério da Saúde. Secretaria de Vigilância em Saúde. Departamento de DST, AIDS e Hepatites Virais (2013). Boletim epidemiológico AIDS/DST. Brasília: Autor.

Moscovici, S. (1978). A representação social: um conceito perdido. In S. Moscovici, A representação social da psicanálise (pp. 41-81). Rio de Janeiro: Zahar.

Moscovici S. (2003). O fenômeno das representações sociais. In S. Moscovici, Representações sociais: investigações em psicologia social (pp. 29-88). Petrópolis: Vozes.

Nascimento, E (2003). Desenvolvimento de pesquisa-ação com caminhoneiros de estrada: trabalhando na problematização as questões voltadas à sexualidade, DST/AIDS e drogas. Tese de Doutorado, Programa de Pós-Graduação em Enfermagem, Escola de Enfermagem de Ribeirão Preto, Ribeirão Preto, SP.

Oltramari, L. C. \& Camargo, B. V. (2004). Representações sociais de mulheres profissionais do sexo sobre a AIDS. Estud. Psicol., 9(2), 317-323.

Pinto, A. C. S., Pinheiro, P. N. C., Vieira, N. F. C., \& Alves, M. D. S. (2007). Compreensão da pandemia da AIDS nos últimos 25 anos. DST. Jornal brasileiro de doenças sexualmente transmissiveis, 19(1), 45-50.

Pottes, F. A. (2007). Aids e envelhecimento: características dos casos com idade igual ou maior que 50 anos em Pernambuco, de 1990 a 2000. Revista Brasileira de Epidemiologia, 10(3), 338-351.

Rebello, L. E. F. S., Gomes, R., \& Souza, A. C. B. D. (2011). Homens e a prevenção da AIDS: análise da produção do conhecimento da área da saúde. Interface - Comunicação, Saúde, Educão, 15(36), 67-78.

Rocha, E. M. D. (2008). DST e AIDS em região de fronteiras: um estudo com caminhoneiros no estado de Rondônia. Dissertação de Mestrado, Programa de Pós-Graduação, Universidade de Brasília, Brasília, DF.

Sá, C. P. D. (1995). Representações sociais: o conceito e o estado atual da teoria. In M. J. Spink (Org.), Conhecimento no cotidiano. (pp. 19-45). São Paulo: Brasiliense.

Silva, C. G. M. D. (2002). O Significado de fidelidade e as estratégias para prevenção da AIDS entre homens casados. Revista de Saúde Pública, 36(4), 40-49.

Spink, M. J. P., Medrado, B., Menegon, V. M., Lyra, J., \& Lima, H. (2001). A construção da AIDS-notícia. Cad. Saúde Pública, 17(4), 851-862, 2001.

Sontag, S. (2007). Doença como metáfora, AIDS e suas metáforas. São Paulo: Companhia das Letras.

Teles, S. A., Matos, M. A., Caetano, K. A. A., Costa, L. A., França, D. D. S., Pessoni, S. M. B, Brunini, S. M., \& Martins, R. M. B. (2008). Comportamentos de risco para doenças sexualmente transmissíveis em caminhoneiros no Brasil. Revista Panamericana de Salud Pública, 24(1), 25-30.

Teixeira, T. R. A., Gracie, R., Malta, M. S., \& Bastos, F. I. (2014). Social geography of AIDS in Brazil: identifying patterns of regional inequalities. Cadernos Saúde Pública, 30(2), 259-271.

The Joint United Nations Programe on HIV/AIDS (Unaids) \& World Health Organization (WHO). (2009). Global summary of the AIDS epidemic, December 2008. In UNAIDS \& WHO. AIDS epidemic update, November. Geneva: Autor.
The Joint United Nations Programe on HIV/AIDS (Unaids). (2007). Prioritized HIV Prevention Measures for Key Audiences. In Unaids. Practical Guidelines for Intensifying HIV Prevention. Geneva: Unaids.

Thiengo, M. A., Oliveira, D. C. D., \& Rodrigues, B. M. R. D. (2005). Representações sociais do HIV/AIDS entre adolescentes: implicações para os cuidados de enfermagem. Revista da Escola de Enfermagem da USP, 39(1), 68-76.

Torres, T. D. L. \& Camargo, B. V. (2008). Representações sociais da Aids e da terapia-retroviral para pessoas vivendo com HIV. Psicologia: Teoria e Prática; 10(1), 64-78.

Villarinho, L. Bezerra, I., Lacerda, R., Latorre, M. R. D. O., Paiva, V., Stall, R., \& Hearst, N. (2002). Caminhoneiros de rota curta e sua vulnerabilidade ao HIV, Santos, SP. Revista de Saúde Pública, 36(4), 61-67.

\section{Agradecimentos}

Aos nossos colaboradores caminhoneiros pela contribuição na pesquisa e por batalharem diariamente por um país melhor. Ao Instituto Multidisciplinar em Saúde (IMS/UFBA) em Vitória da Conquista-BA por ter contribuído com a nossa formação.

Submissão em: 25/12/2012

Revisão em: 01/04/2014

Aceite em: 24/04/2014

Laio Magno Santos de Sousa é Enfermeiro, mestre em Saúde Comunitária pelo Instituto de Saúde Coletiva da Universidade Federal da Bahia (ISC-UFBA), na área de concentração "Ciências Sociais em Saúde". Doutorando do ISC-UFBA na área de "Epidemiologia". Endereço: Rua Basílio da Gama, s/n - Campus Universitário Canela. Salvador/ BA, Brasil. CEP 40.110-040. E-mail: laio_magno@yahoo.com.br

Lucineide Santos Silva é enfermeira, mestre em Enfermagem pela UFBA. Docente da Universidade Federal do Vale do São Francisco, Petrolina/PE, Brasil. E-mail: enflucineide@hotmail.com

Aline Tonheiro Palmeira é Psicóloga, mestre em Psicologia pela UFBA, na linha de pesquisa "Cognição e Representações Sociais". Doutoranda pelo Instituto de Saúde Coletiva/ UFBA na área de concentração "Ciências Sociais em Saúde". E-mail:alinepalmeira@gmail.com

\section{Como citar:}

Sousa, L. M. S., Silva, L. S., \& Palmeira, A. T. (2014). Representações sociais de caminhoneiros de rota curta sobre HIV/AIDS. Psicologia \& Sociedade, 26(2), 346355 . 